\title{
Collective Helping and Bystander Effects in Coevolving Helping Networks
}

\author{
Hang-Hyun Jo, ${ }^{1}$ Hyun Keun Lee, ${ }^{1,2}$ and Hyunggyu Park ${ }^{1}$ \\ ${ }^{1}$ School of Physics, Korea Institute for Advanced Study, Seoul 130-722, Korea \\ ${ }^{2}$ Department of Physics, BK21 Physics Research Division, \\ Sungkyunkwan University, Suwon 440-746, Korea
}

(Dated: June 9, 2018)

\begin{abstract}
We study collective helping behavior and bystander effects in a coevolving helping network model. A node and a link of the network represents an agent who renders or receives help and a friendly relation between agents, respectively. A helping trial of an agent depends on relations with other involved agents and its result (success or failure) updates the relation between the helper and the recipient. We study the network link dynamics and its steady states analytically and numerically. The full phase diagram is presented with various kinds of active and inactive phases and the nature of phase transitions are explored. We find various interesting bystander effects, consistent with the field study results, of which the underlying mechanism is proposed.
\end{abstract}

\section{INTRODUCTION}

In recent years, statistical mechanics has played an important role in quantitative investigation of the social, economic, and psychological phenomena [1]. Some social phenomena bear the resemblance to the physical processes in a sense that the macroscopic complex patterns emerge from the interaction of a large number of microscopic constituents. Along this line, one of the interesting subjects to physicists is opinion dynamics, most of which are concerned either with agent based model studies 2, 3] or with the analysis of real data such as election results 4, 5]. For the model studies the effect of interaction structure has been considered in terms of the complex network theory [6]. More recently, various coevolving networks with the agents interacting on those networks have been proposed and studied [7]. The coevolution of networks with agents may be justified for a broad range of social phenomena, because each agent may construct or reconstruct his or her own neighborhoods in response to the interaction with other agents.

In this paper, we study a coevolving helping network model, known as the rescue model [8]. This model was motivated by the bystander effect observed in social psychology: The witnesses are less likely to intervene in the emergency situation when there are more witnesses. The bystander effect was introduced by Latané and his colleagues in the late 1960's [9]. Since then, the prosocial and helping behavior was investigated extensively from micro- to macro-level perspectives [10]. The meso-level perspective corresponds to the study of helper-recipient dyads. We would like to see how the collective helping behavior at the level of society emerges from the repeated helper-recipient interactions. We adopt the coevolutionary dynamics consisting of two stages such as (a) an agent's behavior depending on the relations with other involved agents and (b) the relation update resulting from the agent's behavior.

The assumptions of the model are based on the results of social psychological experiments in the laboratory $[9$, 11]: First, the witnesses who had an acquaintance with the victim were faster to report the victim's distress than did the other witnesses. Second, the witnesses who had friends among themselves responded to the emergency situation faster. Thus the relations among the involved agents definitely affected their helping behaviors. For simplicity, we assume that the various relations among agents, such as friendship or short acquaintances, can be modeled by a simple (unweighted and undirected) link between nodes of a network, where a node represents an agent.

We interpret the link as one node's expectation of help by the other node. The link density of a network can be interpreted as the aggregate expectation of help in a society. A new link between the intervening witness (helper) and the victim (recipient) may be created as a result of the successful intervention. Or the existing link between them may be severed when the intervention fails, because the failure may reduce the future expectation of help between each other. Furthermore, to build the feasible model we adopt the cost-reward model for the witness's arousal to the emergency [12]. One of the basic assumptions is that the degree of arousal that a witness perceives is a monotonically increasing function of the perceived severity and clarity of the emergency. Here we will use the more explicit term, i.e. the degree of willingness to intervene, rather than the degree of arousal.

In the previous works on the rescue model [8], the authors investigated the effect of the number of involved witnesses $k$ on the collective helping behavior. They reported a non-monotonic variation of the link density (aggregate expectation of help) with $k$ at some moderate values of the model parameters, which are partly consistent with the field study results [13]. In this work, we present the full phase diagram through the comprehensive analytic study of the model with small $k$ in the whole parameter space. We identify the various kinds of active and inactive phases and analyze the nature of phase transitions. Furthermore, we study the fluctuations which are responsible for finite-size effects and correlations. Numerical simulation results for large $k$ reveal various kinds (normal, reverse, complex) of bystander effects depending on the parameter values. We propose the 
underlying mechanism for these bystander effects, which is consistent with the numerical and analytical results.

This paper is organized as following. In Sec. III the coevolving helping network model is briefly introduced. In Sec. III the active and inactive phases and the transitions among them are identified by the analytic calculations and confirmed by numerical simulations. In Sec. IV] the various bystander effects are discussed. Finally, we summarize the results in Sec. V.

\section{MODEL}

We briefly introduce the coevolving helping network (CHN) model [8]. In the CHN, a node represents an agent and a link between two nodes represents a friendly relation between two agents. The links are unweighted and undirected, so the network is defined by the symmetric adjacency matrix $\left\{\rho_{i j}\right\}$ where $\rho_{i j}=1$ if two nodes $i$ and $j$ are connected by a link and 0 otherwise.

At each time step, an accident (emergency) occurs involving a randomly chosen agent $v$ (victim) and also randomly chosen $k$ agents (witnesses) from a population of $N$ agents (nodes). The set of $k$ agents for the victim $v$ is denoted by $\Lambda_{v}$. Each accident carries the degree of its severity represented by $q$, which is randomly drawn uniformly from $[0,1)$. Each witness $i \in \Lambda_{v}$ needs to overcome, at least, its nonnegative potential cost $c_{i}$ to intervene in an accident (rescue attempt). Furthermore, the possibility of the rescue attempt by the witness should be enhanced by the friendly relation between the victim and the witness as well as the number of friends of the witness in the other $k-1$ witnesses.

Summing up all together, we may write the degree of willingness $x_{i v}$ of the witness $i$ to intervene in the accident occurred at time $t$ on the victim $v$ as

$$
x_{i v}(t)=q+a \rho_{i v}(t)+b \sum_{j \in \Lambda_{v}^{i}}\left[2 \rho_{i j}(t)-1\right]-c_{i},
$$

where $a \geq 0, b \geq 0$, and $\Lambda_{v}^{i}$ denotes the set $\Lambda_{v}$ excluding the witness $i$. The interaction term between witnesses enhances $x_{i v}$ if the witness $i$ has more friends than strangers in $\Lambda_{v}^{i}$, and vice versa. The magnitude of the interaction term increases with $k$ as it can vary from $b(k-1)$ (all other witnesses are friends of the witness $i)$ to $-b(k-1)$ (all other witnesses are strangers to the witness $i$ ). So we expect that the interaction term may dominate the evolving dynamics over all other terms for sufficiently large $k$.

We select the witness $i \in \Lambda_{v}$ with the largest value of $x_{i v}$ as the intervener [8]. If there are more than one witnesses with the largest $x_{i v}$, one of them is selected randomly. The intervention attempt would succeed if $x_{i v} \geq 0$ and fail otherwise, which is followed by the link (relation) update between the victim and the intervener. If successful, the friendly relation will be set up or maintained between the victim and the intervener. Otherwise, they become or remain strangers to each other. The link update dynamics is summarized as

$$
\rho_{i v}(t+\Delta t)=\theta\left[x_{i v}(t)\right]
$$

where $\theta(x)$ is a Heaviside step function with $\theta(x)=1$ for $x \geq 0$ and 0 for $x<0$. We choose the time step $\Delta t=1 / L$ for convenience where $L=\frac{N(N-1)}{2}$ is the total number of possible links between $N$ nodes. At the next time step, the above procedure is repeated and the time $t$ is incremented by 1 after $L$ such iterations.

We focus on the link density $\rho(t)$ and the hole density $u(t)$ as the order parameters, respectively:

$$
\rho(t) \equiv 1-u(t) \equiv \frac{1}{L} \sum_{i<j} \rho_{i j}(t),
$$

which should be proportional to the aggregate mean expectation of help (intervention) in a society, which can be one of the important social features.

Finally, in this work, we assume $c_{i}=c \geq 0$ for all $i$ for simplicity, which may be valid for a sufficiently homogeneous population.

\section{NONEQUILIBRIUM PHASE TRANSITIONS}

\section{A. The case with $a=0$ and $b=0$}

The $a=b=0$ case is special. Every witness's degree of willingness is identical as $x_{i v}=q-c$ and the intervener is randomly selected among witnesses, regardless of the number of witnesses $k$. The probability for the successful intervention $\left(x_{i v} \geq 0\right)$ should be equal to $1-c$ for $0 \leq c \leq$ 1. Thus, probabilistically, the number of links increases by 1 with the rate $W_{+}=(1-c)(1-\rho)$ and decreases by 1 with the rate $W_{-}=c \rho$. For $c>1, W_{+}=0$ and $W_{-}=\rho$.

In the mean-field (MF) framework ignoring the stochastic temporal noise, the rate equation for the link density in the time-continuum limit is given as

$$
\frac{d \rho}{d t}=W_{+}-W_{-}
$$

where

$$
\begin{aligned}
& W_{+}=F(1-c)(1-\rho), \\
& W_{-}=F(c) \rho,
\end{aligned}
$$

with $F(x)=x$ for $0<x<1,1$ for $x \geq 1$, and 0 for $x \leq 0$. It is easy to show that $\rho(t)$ behaves as

$$
\rho(t)=\rho(0) e^{-t}+\rho_{\infty}\left(1-e^{-t}\right),
$$

with the steady-state density $\rho_{\infty} \equiv \lim _{t \rightarrow \infty} \rho(t)$ as

$$
\rho_{\infty}=F(1-c)
$$

which implies that there is a nonequilibrium phase transition at $c=1$ from an active phase $\left(\rho_{\infty}=1-c\right.$ for 
$c<1)$ into an inactive phase $\left(\rho_{\infty}=0\right.$ for $\left.c \geq 1\right)$. The order parameter exponent $\beta$ is defined near the transition point in the active side as

$$
\rho_{\infty} \simeq \varepsilon^{\beta},
$$

with the reduced coupling constant $\varepsilon$ which measures the distance from the transition point. In this case, $\varepsilon=1-c$ and $\beta=1$.

In the inactive phase, the steady state is the vacuum (no link state) where the dynamics becomes completely dead, which is called as an absorbing (trapped) state. However, this transition is different from other well-known absorbing phase transitions [15], since the vacuum is not absorbing in the active phase. Furthermore, the system at the transition point $(c=1)$ is not critical, but absorbing: The link density decays exponentially $\left(\rho(t)=\rho(0) e^{-t}\right)$.

One may derive the exact Langevin equation for large $L$, including the stochastic noise (in the Ito sense [16]) through the usual Fokker-Planck formulation as

$$
\frac{d \rho}{d t}=f(\rho)+\sqrt{g(\rho)} \xi(t)
$$

where $f(\rho)=W_{+}-W_{-}$and $g(\rho)=\left(W_{+}+W_{-}\right) / L$. $\xi(t)$ is a white noise with zero mean satisfying $\left\langle\xi(t) \xi\left(t^{\prime}\right)\right\rangle=\delta(t-$ $\left.t^{\prime}\right)$ where $\langle\cdots\rangle$ is the noise average. The drift function $f(\rho)=(1-c)-\rho$ and the multiplicative factor $g(\rho)=$ $[(1-c)+(2 c-1) \rho] / L$ for $c<1$, while $f(\rho)=-\rho$ and $g(\rho)=\rho / L$ for $c \geq 1$. In the $L=\infty$ limit, the noise term becomes negiligible and Eq. (4) becomes exact.

The fluctuations due to the stochastic noise can be derived, using the Ito calculus [16] such that

$$
\frac{d\langle G(\rho)\rangle}{d t}=\left\langle f(\rho) \frac{\partial G}{\partial \rho}\right\rangle+\frac{1}{2}\left\langle g(\rho) \frac{\partial^{2} G}{\partial \rho^{2}}\right\rangle,
$$

where $G$ is a general function of $\rho$. Then, the order parameter fluctuation $\chi$ defined as

$$
\chi=L\left[\left\langle\rho^{2}\right\rangle-\langle\rho\rangle^{2}\right],
$$

behaves in the steady state as

$$
\chi_{\infty}=\left\{\begin{array}{cc}
c(1-c) & \text { for } c<1 \\
0 & \text { for } c \geq 1
\end{array} .\right.
$$

Note that the fluctuation vanishes as the system approaches the transition point $(c=1)$. The fluctuation exponent $\gamma^{\prime}$ defined as $\chi \sim \varepsilon^{-\gamma^{\prime}}$ is given as $\gamma^{\prime}=-1$.

\section{B. The case with $a>0$ and $b=0$}

For $a>0$ and $b=0$, we have the same rate equation as in Eq. (4) with the link creation and annihilation rates as

$$
\begin{aligned}
& W_{+}=F(1-c)(1-\rho)^{k}, \\
& W_{-}=F(c-a)\left[1-(1-\rho)^{k}\right],
\end{aligned}
$$

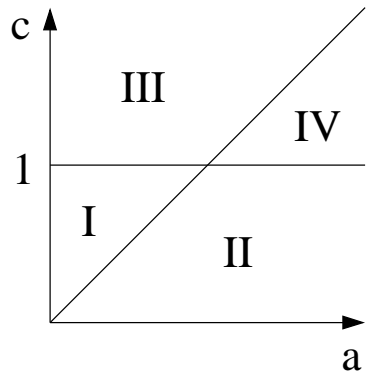

FIG. 1: The phase diagram for the case with $b=0$. The parameter space $(a, c)$ is divided into four regions by two transition lines of $c=1$ and $c=a$.

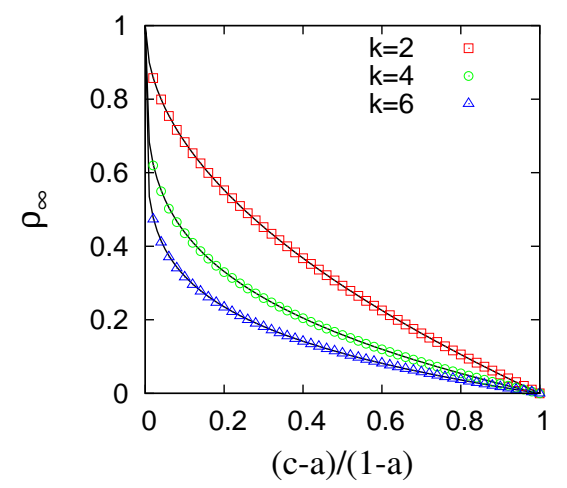

FIG. 2: (Color online) The steady-state link density $\rho_{\infty}$ along the $a=0.5$ line for $k=2,4$, and 6 .

respectively. Note that the $a=0^{+}$limit is singular except the case of $k=1$.

It is straightforward to analyze the rate equation, which yields the steady-state density and its long-time dynamics. The parameter space $(a, c)$ is divided into four different regions separated by two transition lines of $c=1$ and $c=a$ (Fig. 11). In region I $(a<c<1)$, we have a finite link density as

$$
\rho_{\infty}=1-[(c-a) /(1-a)]^{1 / k}
$$

(active phase). In region II $(c<a$ and $c<1)$, we find the fully connected network; $\rho_{\infty}=1$ (paradise phase). In region III $(c>a$ and $c>1)$, the network becomes completely disconnected; $\rho_{\infty}=0$ (isolation phase). In region IV $(1<c<a)$, both transition rates $W_{+}=W_{-}=$ 0 and no dynamics occurs; $\rho(t)=\rho(0)$. Of course, the region IV is unphysical, so we focus on the three other regions and the transition lines between them.

Approaching the I-III transition line $\left(c=1^{-}\right)$inside the active phase, the order parameter scales as

$$
\rho_{\infty} \simeq A_{1} \varepsilon_{1}^{\beta_{1}},
$$

with $A_{1}=1 /[k(1-a)], \varepsilon_{1}=1-c$, and $\beta_{1}=1$. Near the I-II transition line $\left(c=a^{+}\right)$, the hole density $u=1-\rho$ plays a role of the order parameter, which scales as

$$
u_{\infty} \simeq A_{2} \varepsilon_{2}^{\beta_{2}},
$$


with $A_{2}=(1-a)^{-1 / k}, \varepsilon_{2}=c-a$, and $\beta_{2}=1 / k$.

Note that the steady-state link density $\rho_{\infty}$ becomes smaller as $k$ increases. In fact, one can easily show from Eq. (14) that this holds for the whole region of the active phase, see Fig. 2. It implies that the society becomes less friendly (lower link density) with large $k$ (more crowded society like big cities) in the long-time limit (normal bystander effect). Therefore, one may regard the normal bystander effect as the result of iterative intervention attempts at the level of society.

From the dynamic rules (transition rates) of our model, Eq. (13), it is easy to understand how this happens. A new link may be created only when there are no existing links between a victim and any of $k$ witnesses, which we call the $k$-hole constraint. (Note that this applies only when $a>0$.) The probability of finding this situation is proportional to $(1-\rho)^{k}$, which monotonically decreases with $k$. So one can expect that the steady-state link density should be smaller with larger $k$. However, with interactions between witnesses $(b \neq 0)$, the $k$-hole constraint is not absolutely necessary to create a new link, which will be discussed later.

Furthermore, suppose we are allowed to control $c$, for example, decrease $c$ by giving all agents the same incentive for intervention. The efficiency of our policy might be measured by the slope of the link density, i.e. $e \equiv-\frac{\partial \rho}{\partial c}$. As can be easily seen in Fig. 2, the policy becomes quite effective near the paradise phase and more effective for larger $k$. However, it will be less efficient near the isolation phase and much lesser for larger $k$. These results imply that the incentive policy would not work well in unfriendly and crowded communities like big and rapidly developed cities, but may work efficiently in friendly and crowded communities like a guild.

Now, we study the long-time dynamics in various regions. In the regions I and III, the link density decays exponentially: $\rho(t)-\rho_{\infty} \sim e^{-t / \tau}$ with the characteristic time $\tau$ such that $\tau^{-1}=k(1-a)^{1 / k}(c-a)^{1-1 / k}$ in the active phase and $\tau^{-1}=k(c-a)$ for $c<a+1$ or $k$ for $c \geq a+1$, respectively in the isolation phase. Along the I-III transition line, the link density still decays exponentially with $\tau^{-1}=k(1-a)$, which implies that this transition line is not critical but absorbing, i.e. the $c=1$ line belongs to the isolation phase. Approaching the I-II transition line $\left(c=a^{+}\right)$, the relaxation time diverges as $\tau \sim \varepsilon_{2}^{-\nu_{\|}}$with the relaxation time exponent $\nu_{\|}=1-1 / k$ for $k>1$. So the incentive policy works efficiently in this region, but its effect will show up quite slowly.

In the region II (paradise phase), the long-time decay dynamics follows a much slower power-law: the hole density $u(t) \sim t^{-\delta}$ with the decay exponent $\delta=1 /(k-1)$ for $k>1$ except that $u(t) \sim e^{-(1-c) t}$ for $k=1$. With larger $k$, the dynamics becomes extremely slower. The I-II transition line belongs to the paradise phase, meaning that the system exhibits the same power-law decay dynamics.

As in Eq. (9), one may write the exact Langevin equation including the stochastic noise with the transition rates given by Eq. (13). Near the I-III transition line, one can expand in powers of $\rho$ and obtain that $f(\rho)=$ $(1-c)-(1-a) k \rho$ and $g(\rho)=[(1-c)+(2 c-a-1) k \rho] / L$ in the active phase, while $f(\rho)=-(c-a) k \rho$ and $g(\rho)=$ $(c-a) k \rho / L(c<a+1)$ or $k \rho / L(c \geq a+1)$ in the isolation phase. Then, the steady-state fluctuations are analytically obtained, similar to the last subsection, as $\chi_{\infty} \simeq B_{1} \varepsilon_{1}^{-\gamma_{1}^{\prime}}$ with $B_{1}=(c-a) /\left[k(1-a)^{2}\right]$ and $\gamma_{1}^{\prime}=-1$ in the active side and $\chi_{\infty}=0$ in the isolation side.

Near the I-II transition line, we need to write the Langevin equation in terms of the hole density $u$ as

$$
\frac{d u}{d t}=\tilde{f}(u)+\sqrt{\tilde{g}(u)} \xi(t),
$$

where $\tilde{f}(u)=(c-a)-(1-a) u^{k}$ and $\tilde{g}(u)=[(c-a)+(1+$ $\left.a-2 c) u^{k}\right] / L$ in the active phase, and $\tilde{f}(u)=-(1-c) u^{k}$ and $\tilde{g}(u)=(1-c) u^{k} / L$ in the paradise phase.

One can derive the $k$-th order fluctuation analytically, using the Ito calculus of Eq. (10) as

$$
\chi_{\infty}^{(k)} \equiv L\left[\left\langle u^{k+1}\right\rangle-\left\langle u^{k}\right\rangle\langle u\rangle\right]=B_{2} \varepsilon_{2}^{-\gamma_{2}^{\prime(k)}},
$$

where $B_{2}=(1-c) /(1-a)^{2}$ and $\gamma_{2}^{\prime(k)}=-1$. From the simple scaling theory, one can relate the ordinary mean-square fluctuation $\chi_{\infty}=\chi_{\infty}^{(1)}$ with $\chi_{\infty}^{(k)}$ through the exponent relation of $\gamma_{2}^{\prime}=\gamma_{2}^{\prime(k)}+(k-1) \beta_{2}$. Hence, we find $\gamma_{2}^{\prime}=-1 / k$ with $\beta_{2}=1 / k$.

Finally, we discuss the finite-size effects near the III transition line. The standard finite-size-scaling (FSS) theory predicts

$$
\chi_{\infty}^{(k)}=\varepsilon_{2}^{-\gamma_{2}^{\prime(k)}} \phi^{(k)}\left(\varepsilon_{2} L^{1 / \bar{\nu}_{2}}\right),
$$

where the scaling function behaves as $\phi^{(k)}(x) \sim O(1)$ for large $x$ and $\phi(x)^{(k)} \sim x^{\gamma_{2}^{\prime(k)}}$ for small $x$. Fig. 3)(a) shows an excellent collapse of various numerical finite-size data for $\chi_{\infty}^{(2)}$ at $k=2$ and $a=1 / 2$ onto a scaling curve $\phi^{(2)}(x)$, with the choice of the FSS exponent $\bar{\nu}_{2}=1$. The same is true for the ordinary fluctuation $\chi_{\infty}$ with the scaling function $\phi(x)$ which behaves as $\phi(x) \sim O(1)$ for large $x$ and $\phi(x) \sim x^{\gamma_{2}^{\prime}}$ for small $x$ (Fig $3(\mathrm{~b})$ ). It follows that the order parameter $u$ should scale as $u \sim L^{-\beta_{2} / \bar{\nu}_{2}}=L^{-1 / 2}$ at $\varepsilon_{2}=0^{+}$. Note that the FSS exponent $\bar{\nu}_{2}$ does not satisfy the standard hyperscaling relation as $\gamma_{2}^{\prime}+2 \beta_{2}=$ $\bar{\nu}_{2}$.

In the paradise phase including the I-II boundary, only the link creation process is allowed (no link annihilation dynamics). So there can be infinitely many absorbing states where each node has some unconnected links (holes) and the number of those holes is less than $k$ for all nodes. Then, as there are no configurations satisfying the $k$-hole constraint at all nodes, a link creation attempt is impossible and the system is frozen dynamically. The hole density in such absorbing states should be proportional to $k N / L$ or equivalently $u \sim L^{-1 / 2}$ in this region 

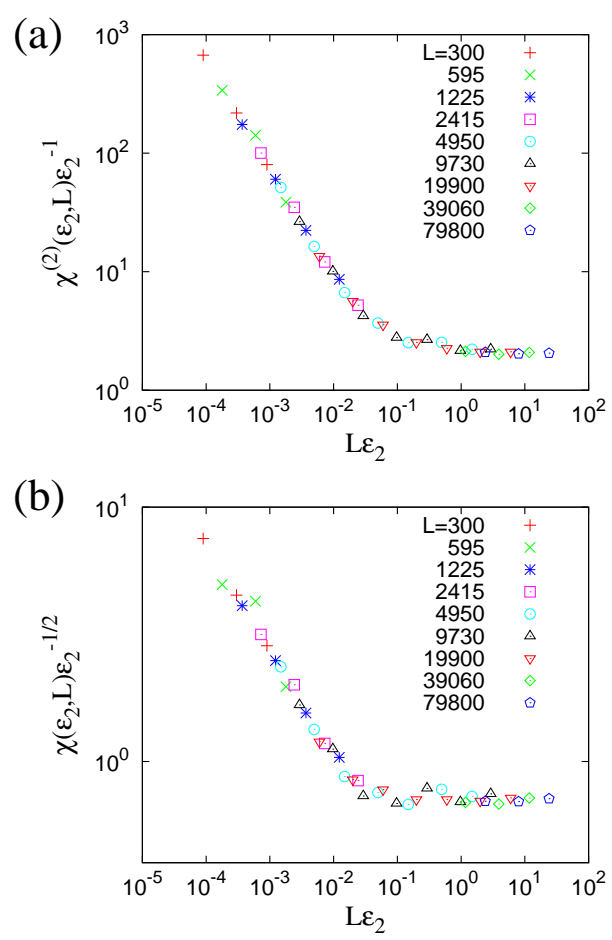

FIG. 3: (Color online) The scaling collapse of numerical data for the fluctuations (a) $\chi_{\infty}^{(2)}$ and (b) $\chi_{\infty}$ at $k=2$ and $a=0.5$ in the active phase $\left(\varepsilon_{2}>0\right)$.

$\left(\varepsilon_{2} \leq 0\right)$, which is also confirmed numerically. In addition, there is a discontinuous jump in the hole density for finite systems across the I-II transition line, due to the presence of this frozen dynamics in the paradise phase.

\section{The case with $b>0$}

We now consider more realistic cases with the interaction term $(b>0)$. In this case, each witness's degree of willingness in Eq. (11) is enhanced if he has more friends than strangers among the other witnesses and vice versa. Then, one may naively expect that the interaction drives a high link-density network to become a higher one, and a low link-density network to become a lower one. As a result, both the isolation phase and the paradise phase would expand into the active phase. This is true at $a=0$ and, for sufficiently large $b(\geq 1 /[2(k-1)])$, the active phase squeezes down to disappear (see, for example, Fig. (4).

However, for $a>0$, the $k$-hole constraint is in effect, which suppresses the link density increase in general. Its effect is particularly big in the high density networks, so the paradise phase may shrink. At the same time, the interaction term may loosen the constraint for large $k$ and thus the naive picture can be restored. In fact, the $k$-hole constraint becomes loose for $a<2 b(k-2)$, when one of the witnesses without a link to the victim may

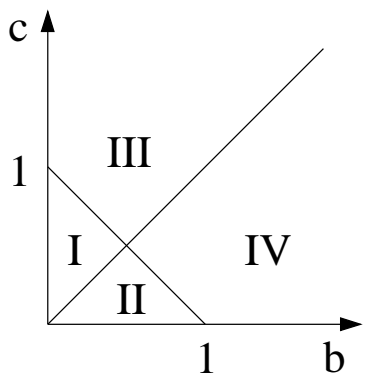

FIG. 4: The phase diagram for the case with $a=0$ and $k=2$.

take a higher degree of willingness than one with a link to the victim. So, a link creation dynamics is possible for a configuration not satisfying the $k$-hole constraint.

At $k=2$, the $k$-hole constraint remains intact for any value of $b$ and $a>0$. The link density decreases even in the high link-density networks. Then, the active phase expands into the paradise phase and also extends to the large $a$ region. In contrast, for $k \geq 3$ and small $a$ (< $2 b(k-2))$, we find that the naive expectation holds and the active phase shrinks due to the expansion of both the isolation and the paradise phase.

As discussed in the last subsection for $b=0$, the $k$ hole constraint is the key factor for the normal bystander effect: the steady-state link density $\rho_{\infty}$ decreases monotonically with $k$. For $b>0$, however, as $k$ increases, the constraint becomes looser and the interaction term becomes stronger $(\sim b(k-1)(2 \rho-1))$ in the high density regime $(\rho>1 / 2)$. In this case, we expect the density increase with $k$ for sufficiently large $k$ after the ordinary density decrease with $k$ for small $k$. These non-monotonic bystander effects will be discussed more in the next section.

Now, we consider the $k=2$ and $k \geq 3$ case in more details.

$$
\text { 1. } k=2
$$

First, consider the $a=0$ case. One may easily show that the transition rates are given as

$$
\begin{aligned}
& W_{+}=(1-\rho)[F(1-c-b)(1-\rho)+F(1-c+b) \rho] \\
& W_{-}=\rho[F(c+b)(1-\rho)+F(c-b) \rho]
\end{aligned}
$$

respectively. We find the phase diagram as in Fig. 4 where the four different phases are separated by two transition lines of $c=b$ and $c=1-b$. As expected, the active phase shrinks with increasing $b$ by invasion of both the isolation and the paradise phase. In the active phase, the steady-state link density is $\rho_{\infty}=(1-b-c) /(1-2 b)$, which scales linearly $\left(\beta_{1}=1\right)$ near the I-III transition line $(c=1-b)$ and the hole density $u_{\infty}$ also scales linearly $\left(\beta_{2}=1\right)$ near the I-II transition line $(c=b)$.

With finite $a>0$, the $k$-hole constraint is in effect and 


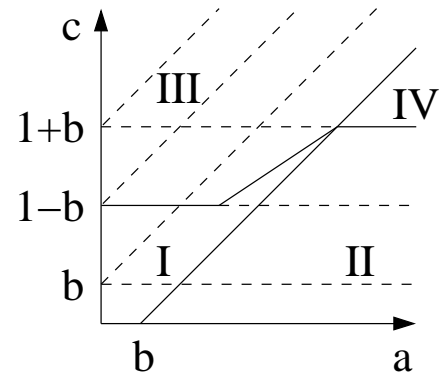

FIG. 5: The phase diagram for the case with $k=2$ and $0<$ $b<\frac{1}{2}$. The parameter space $(a, c)$ is divided by four transition lines such as $c=1 \pm b, c=a-b$, and $c=(1+2 a-b) / 3$. The dashed lines are 7 non-analytic lines for the transition rates.

the transition rates are modified as

$$
\begin{aligned}
& W_{+}=(1-\rho)^{2}[F(1-c-b)(1-\rho)+F(1-c+b) \rho], \\
& W_{-}=\rho(2-\rho)[F(c-a+b)(1-\rho)+F(c-a-b) \rho],
\end{aligned}
$$

respectively. Note that the $a=0^{+}$limit is again singular due to the $k$-hole constraint.

The parameter space $(a, c)$ is divided into 14 different regions by 7 lines such as $c=1 \pm b, c=b, c=a+(1 \pm b)$, and $c=a \pm b$, where the transition rates change abruptly due to the non-analyticity of the function $F(x)$ at $x=0$ and 1 . The divided regions are grouped into the four phases as shown in Fig. 5. except that one central region is divided into two phases of I and III for $0<b<1 / 2$. As expected, the link density decreases with $b$ for most regions [17], mainly due to the $k$-hole constraint. The isolation phase III invades the active phase I and the paradise phase II retreats. We find the similar result for $b>1 / 2$ (not shown here).

The active phase I comprises of four sub-phases where the order parameter $\rho_{\infty}$ behaves differently:

$$
\begin{aligned}
& 1-\rho_{\infty}= \\
& \left\{\begin{array}{l}
\frac{b+\sqrt{b^{2}+(1-a)(c-a-b)}}{1-a} \\
\frac{c-a+b}{1+a-2 c} \\
\frac{1-\sqrt{1+4 a^{2}-4 a b-4 a c}}{2 a} \\
\frac{1+b-c-\sqrt{4 a^{2}-3 b^{2}+2 b+1-2(4 a+b+1) c+5 c^{2}}}{2(a+b-c)}
\end{array}\right.
\end{aligned}
$$

All transitions are continuous but not differentiable between the phases of I, II, and III, as well as between the four active sub-phases. In the vicinity of all transition lines, either the link density $\rho_{\infty}$ or the hole density $u_{\infty}$ vanishes linearly $\left(\beta_{1}=\beta_{2}=1\right)$.

It is interesting to note the emergence of a nontrivial phase transition between $\mathrm{Ib}$ and III in the region of $1-b<c<1+b$, where the link creation and the link annihilation dynamics compete each other. In this region, only the process representing the second term of
$W_{+}$in Eq. (21) is possible in the link creation dynamics. Therefore, the link creation dynamics is impossible when $\rho$ becomes zero (no-link state). Once the system gets into the no-link state, it cannot escape out of that state (absorbing state). Based on these observations, we can map the dynamics onto the well-known contact process [18], which exhibits an absorbing phase transition from vacuum (isolation phase) into an active phase. This transition is known to belong to the so-called directed percolation (DP) universality class [15].

Near the transition between Ib and III, we can easily write the Langevin equation for small $\rho$ as

$$
\frac{d \rho}{d t}=3 \varepsilon_{1} \rho-B \rho^{2}+\sqrt{\frac{4 B \rho}{L}} \xi(t),
$$

where $\varepsilon_{1}=c_{1}^{*}-c$ with the transition point $c_{1}^{*}=(1+2 a-$ $b) / 3$ and $B=(1-a+2 b) / 3>0$. This equation is identical to the MF Langevin equation describing the DP-type absorbing phase transition, which is characterized by the noise amplitude proportional to $\sqrt{\rho}$. The steady-state link density behaves as $\rho_{\infty} \simeq(3 / B) \varepsilon_{1}^{\beta_{1}}$ with $\beta_{1}=1$. It is well known that the fluctuation exponent $\gamma_{1}^{\prime}=0$ in the active side for the MF DP universality class [15]. Utilizing the hyperscaling relation, we find the FSS exponent $\bar{\nu}_{1}=2$, and thus expect $\rho_{\infty} \sim L^{-\beta_{1} / \bar{\nu}_{1}}=L^{-1 / 2}$ at the transition [19], which is confirmed numerically (not shown here). The relaxation time also diverges as $\tau \sim \varepsilon_{1}^{-\nu_{\|}}$with $\nu_{\|}=1$, in contrast to the case of the Ia-III transition line where $\tau$ is finite.

The transition from Ib to II is also interesting. The corresponding Langevin equation in the active side is given, in terms of the hole density $u=1-\rho$, as

$$
\frac{d u}{d t}=\varepsilon_{2} u-3 B u^{2}+\sqrt{\frac{6 B u^{2}}{L}} \xi(t),
$$

where $\varepsilon_{2}=c-c_{2}^{*}$ with $c_{2}^{*}=a-b$. The steady-state hole density scales as $u_{\infty} \simeq(1 /(3 B)) \varepsilon_{2}^{\beta_{2}}$ with $\beta_{2}=1$. This type of the multiplicative noise linear in the activity field $u$ has been studied extensively in literatures to describe various physical systems including nonequilibrium wetting and synchronization phenomena for spatially extended systems [20]. Naive power counting which is expected to hold in the MF systems yields the FSS expo- nent $\bar{\nu}_{2}=1$ and $\gamma_{2}^{\prime}=-1$. Thus, in the limit of $\varepsilon_{2}=0^{+}$, we have $u \sim L^{-1}$. As $u \sim L^{-1 / 2}$ in the paradise phase and at the transition $\left(\varepsilon_{2} \leq 0\right)$, we expect a discontinuous jump in the hole density for finite systems at the Ib-II transition. Similar behaviors are found near the transitions from Id and Ic to II. All these results are confirmed numerically (not shown here).

Note that the exponent $\beta_{2}$ changes from $1 / k=1 / 2$ to 1 with the interaction term $(b>0)$. The relaxation time exponent $\nu_{\| \mid}$also changes from $1-1 / k=1 / 2$ to 1. Inside the paradise phase II, the linear term (the first term in the right-hand side of Eq. (24)) vanishes and the hole density decay exponent becomes $\delta=1$, which is the same as $\delta=1 /(k-1)=1$ at $b=0$. The FSS exponent $\bar{\nu}_{2}=1$ for both $b=0$ and $b>0$. 


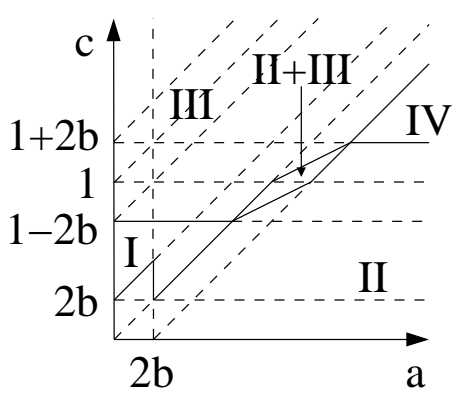

FIG. 6: The phase diagram for the case with $k=3$ and $0<b<\frac{1}{6}$.

\section{2. $k \geq 3$}

For large $k$, we expect more complicated phase diagrams. For example, see Fig. 6 for the phase diagram at $k=3$ and small $b$. The parameter space is divided by 11 lines, such as $c=1 \pm 2 b, c=1, c=2 b, c=a+(1 \pm 2 b)$, $c=a+1, c=a \pm 2 b, c=a$, and $a=2 b$. The last line of $a=2 b$ is the boundary line between regions where the $k$-hole constraint is strictly valid and becomes loose. The divided regions are grouped into five phases including one new phase (inactive 'mixed' phase) in the central region (Fig. 6).

In this mixed phase, the system reaches either the isolation phase or the paradise phase, depending on the initial conditions and the stochastic dynamics. This is similar to the absorbing phase belonging to the directed Ising universality class [21] with two symmetric absorbing states. But, here, there is no symmetry between absorbing states.

Our main interest lies in the active phase and the surrounding inactive (isolation and paradise) phases. As expected, we find that the active phase shrinks due to the expansion of both inactive phases. But, the expansion of the paradise phase is both qualitatively and quantitatively different in the regions of $a>2 b$ and $a<2 b$ at $k=3$. For $a>2 b$, the $k$-hole constraint remains intact. The density decrease due to the $k$-hole constraint happens to be balanced exactly by the density increase due to the interactions at $k=3$, which results in maintaining the I-II phase boundary $(c=a)$ as it is at $b=0$. For $k>3$, the interaction term dominates and thus the paradise phase invades into the active phase. For $a<2 b$ where the $k$-hole constraint is loose, the active phase shrinks more and the I-II phase boundary is given by the $c=a+2 b$ line.

The hole density in the phase II decays as $u(t) \sim t^{-\delta}$ with $\delta=1 / 2$ for $a>2 b$ and $\delta=1$ for $a<2 b$. For general $k$, we find $\delta=1 /[k / 2]([x]$ is the integer value of $x)$ for $a<2 b$. Extending to the other regions of $2 b(n-1)<$ $a<2 b n$ with an integer $n \leq k-2$ is straightforward (not reported here). For $a>2 b(k-2)$ where the $k$-hole constraint is valid, we find $\delta=1 /(k-1)$ (the same as that at $b=0$ ).
Finally, we report the values of scaling exponents near the I-II boundary. We find $\beta_{2}=1, \bar{\nu}_{2}=1, \nu_{\|}=2$ for $a<2 b$ and $\beta_{2}=1 / 2, \bar{\nu}_{2}=1 / 2, \nu_{\|}=1 / 2$ for $a>2 b$. Generalization to higher $k$ is straightforward. Near the I-III boundary, we find $\beta_{1}=1$, which holds for any $k$.

\section{BYSTANDER EFFECTS AND SOCIAL IMPLICATIONS}

We investigate various bystander effects at the level of society/community for general $k$, in relation to the empirical data analysis by social psychologists. Our model is set up, based on the experimental results in the laboratory, but the specific values of control parameters cannot be directly inferred from these results. We compare our model study with corresponding field studies [13, 14], in order to understand its social implications properly.

The control parameters $(a, b, c, k)$ can be regarded as given or intrinsic to a society/community, hence depending on various factors of its socioeconomic condition and also on the taxonomy of helping behavior as well [13, 14]. For the taxonomy of helping, social psychologists introduced three independent dimensions: doing (direct help) versus giving (indirect help), spontaneous versus planned, and serious versus nonserious. Helping measures, such as helping rate, were observed from field studies performed in the streets of 55 cities and towns in Australia [13] and of 36 cities in U.S. [14].

For example, in one of experiments the experimenter walking on a street drops a pen accidentally in front of a solitary pedestrian, and continues walking, then sees whether the pedestrian picks the pen and bring it to the experimenter or simply ignores. The helping rate may correspond to the success rate in our model, which is defined as the number of successful interventions divided by the total number of interventions. In general the success rate is a monotonic function of the link density $\rho$, i.e. the aggregate expectation of help in a community. Therefore we can interpret $\rho$ as a helping measure of a community. The helping behavior in this experiment belongs to the direct, spontaneous, and nonserious type with a low potential cost $(\operatorname{small} c)$, while the rescue attempts in emergency situations are classified into the direct, spontaneous, and serious type with large $c$.

The socioeconomic factors (community variables) include the population size, population density, cost of living, unemployment rates, and etc. Here the number of witnesses $k$ per accident corresponds to the population density (crowdedness) and the system size $N$ to the population size, respectively. While the population density and the population size are highly correlated in reality (big cities are usually crowded), we considered them as being independent to each other for generality.

The previous empirical studies showed that the most relevant factors for the helping rate are the population density and the population size. The strong negative correlation between the helping rate and the population den- 

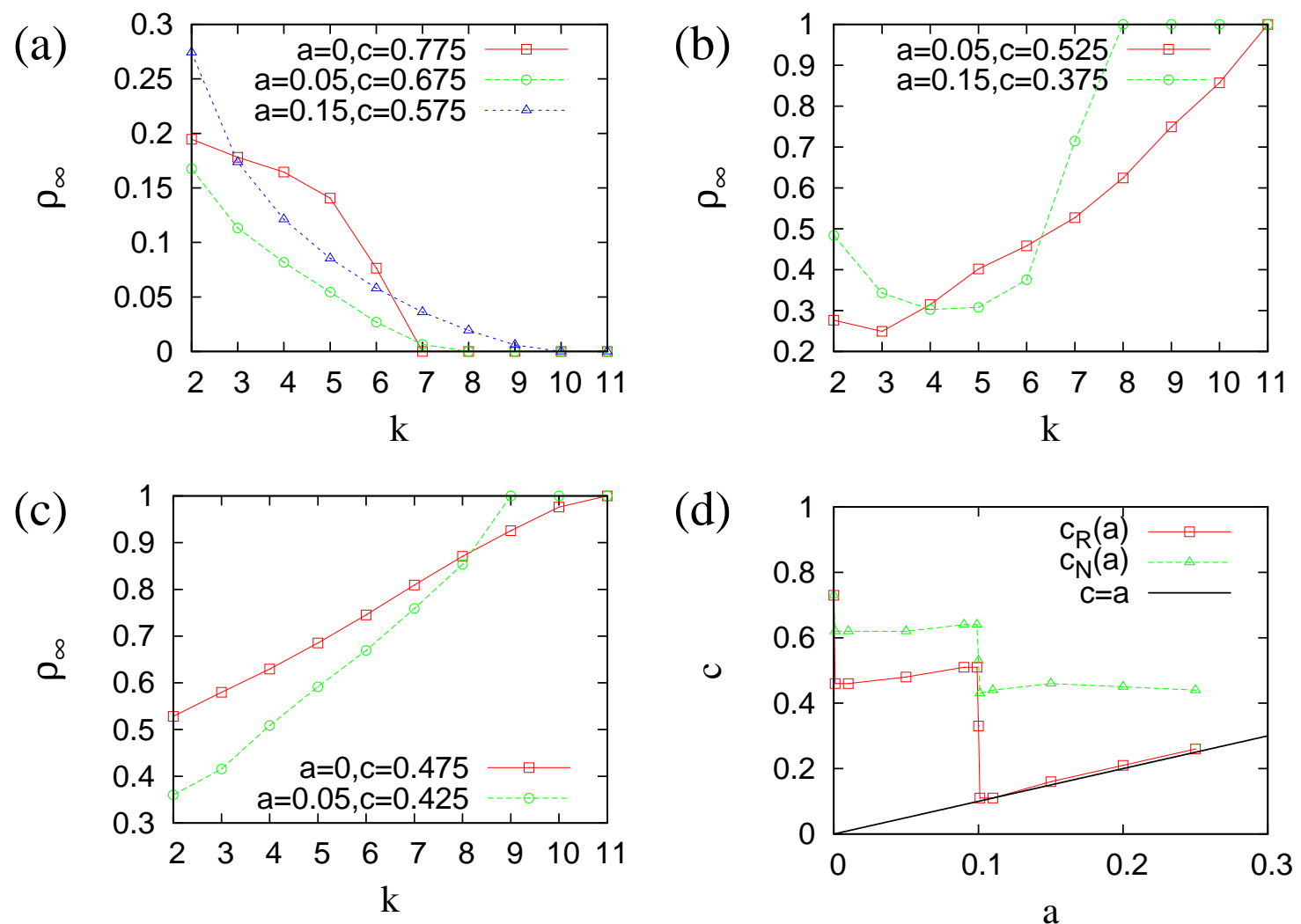

FIG. 7: (Color online) The various bystander effects: (a) NBE, (b) CBE, and (c) RBE when $b=0.05$ and $a=0, b$, and $3 b$. (d) Critical potential cost lines $c_{\mathrm{R}}(a)$ and $c_{\mathrm{N}}(a)$ in the $(a, c)$ phase diagram at fixed $b=0.05$. We find the NBE for $c>c_{\mathrm{N}}(a)$ and the RBE for $c<c_{\mathrm{R}}$. In between these two boundaries, the CBE is found. When identifying the BE's we consider only the active phase, where only one stationary link density exists.

sity/size was found. This implies the normal bystander effect, i.e. $\rho$ decreasing with $k$ in our model. However, in some nonserious experiments, the helping rate first decreases and then increases as the population size (or density) increases [13], and a slightly positive correlation between the helping rate and the population density was also found [14]. These unusual bystander effects can be supported by various behaviors of $\rho$ observed in our model study (see below).

The experiments in the field studies have been done for measuring the aggregate expectation of help in a given society, not for simulating our model dynamics. We assume that the helping network in the society has evolved by our model dynamics and has become already stationary at the time of the experiments. By comparing the experimental data with the stationary results of our model, one may check the validity of our coevolving helping network $(\mathrm{CHN})$ model and also predict some social features in real social systems. Especially, we focus on the various bystander effects found in the field studies.

Now we discuss the $k$-dependent behavior of the link density $\rho$, based on the numerical results for the $\mathrm{CHN}$ model, shown in Fig. 7 The behaviors can be categorized into three: (i) the normal bystander effect (NBE), i.e. $\rho$ decreasing with $k$, (ii) the reverse bystander effect
(RBE), i.e. $\rho$ increasing with $k$, and (iii) the complex bystander effect (CBE), i.e. $\rho$ decreasing and then increasing with $k$. With $b$ fixed, for each value of $a$, there appear two critical values of the potential cost $c$, separating the three different BE's. We observe the NBE phase for $c>c_{\mathrm{N}}(a)$, the CBE phase for $c_{\mathrm{R}}(a)<c<c_{\mathrm{N}}(a)$, and the RBE phase for $c<c_{\mathrm{R}}(a)$, respectively.

To understand how the various BE's appear as the potential cost $c$ varies, we first consider the simplest case of $a=0$ in Eq. (11). For large $c$, the possibility of successful intervention becomes small. This negative effect in the link density $($ small $\rho$ ) is accelerated with $k$, because the $k$-dependent interaction term becomes negative. Therefore, we expect that $\rho$ decreases with $k$ (NBE) in the stationary state. For small $c$, the tendency toward successful intervention is accelerated with $k$ in a positive way. So we expect the RBE.

To be more specific, the dynamics can be simplified by the mean field (MF) approximation of $x_{v i}$ at $a=0$ :

$$
\rho(t+\Delta t)=1+b(k-1)[2 \rho(t)-1]-c,
$$

then the stationarity condition yields

$$
\rho_{\infty}=\frac{1}{2}+\frac{c_{0}-c}{1-2 b(k-1)}
$$


with $c_{0}=1 / 2$. One can easily see the NBE phase for $c>$ $c_{0}$, and the RBE phase for $c<c_{0}$. Numerical simulation results are consistent with this MF picture, except that $c_{0} \simeq 0.73$.

The positive $a$ enhances the possibility of selecting the witness $i$ connected to the victim $\left(\rho_{i v}=1\right)$ as an intervener, and hence the $k$-hole constraint comes into play. The $k$-hole constraint, in general, suppresses the link density $\rho$. So we expect that the NBE phase is not affected, because $a$ only accelerates the decay of $\rho$ with $k$ faster.

However, the RBE phase may be modified significantly in the region where the $k$-hole constraint becomes important. Here, the interaction term proportional to $b(k-1)$ competes with $a$. For sufficiently large $k$, we may ignore $a$ compared to the interaction term, and then $\rho$ increases with $k$. However, for small $k$, the effect of positive $a$ becomes strong so the $k$-hole constraint may make $\rho$ to decrease with $k$ up to some value of $k$. One may expect that this non-monotonic behavior of $\rho$ with $k$ (CBE) appears between the NBE and the RBE phase (Fig. 7(d)).

Note that there is a big jump in the phase boundary at $a=2 b$. As discussed in the previous section, the $k$-hole constraint becomes loose for $a<2 b$, and becomes strong for $a>2 b$. As a result, the link density decreases discontinuously as one crosses the $a=2 b$ point (see, e.g., Fig. 6). So it is easily expected that the NBE phase expands and the RBE phase shrinks. Moreover, the RBE phase effectively disappears for $a>2 b$, because it is almost squeezed down to the $c=a$ line where the paradise phase $(\rho=1)$ starts to appear.

The complex and reverse bystander effects found in our model can support the unusual bystander effects found for the nonserious experiments (small $c$ ). Moreover, when the relation between witnesses is more important than the witness-victim relation ( $a$ is smaller than $b$ ), our model predicts that the $\mathrm{CBE}$ and the $\mathrm{RBE}$ are more commonly observed in nonserious experiments. To confirm the robustness of $\mathrm{CBE}$ and $\mathrm{RBE}$ in reality, more empirical/experimental studies are necessary.

\section{SUMMARY}

We have studied how the collective helping behavior and bystander effects at the level of society emerge from the repeated helper-recipient interactions by means of the coevolving helping network model. Its dynamic rules are based on the results of social psychological experiments. By analyzing the dynamics of the network link density (aggregate expectation of help) and its steady states and fluctuations, we present the full phase diagram with various active and inactive phases and explore the nature of phase transitions between them. Close to the transition between the active and paradise phases, the critical behavior turns out to depend on the number of witnesses per accident $k$. This is due to the link creation suppressed by the $k$-hole constraint, which also governs the algebraic decaying behavior in the paradise phase.

We have numerically found various kinds (normal, reverse, and complex) of collective bystander effects for large $k$ and proposed the underlying mechanism. The normal and complex (non-monotonic) bystander effects are consistent with the field study results for the cases with serious and nonserious helping situations, respectively. In addition, we expect the reverse bystander effect to occur when the witness-victim relation is sufficiently less important than the relation among witnesses for nonserious helping situations.

\section{Acknowledgments}

HJ thanks Eunyoung Moon for helpful discussions.
[1] C. Castellano, S. Fortunato, and V. Loreto, Rev. Mod. Phys. 81, 591 (2009); R.N. Mantegna and H.E. Stanley, An Introduction to Econophysics (Cambridge University Press, Cambridge, England, 2000); Dynamical Systems in Social Psychology, edited by R.R. Vallacher and A. Nowak (Academic, New York, 1994).

[2] D. Stauffer, in Modeling Cooperative Behavior in the Social Sciences, edited by P.L. Garrido, J. Marro, and M.A. Munoz, AIP Conf. Proc. No. 779 (AIP, Melville, NY, 2005), p. 56.; arXiv:0705.0891 1.

[3] K. Sznajd-Weron and J. Sznajd, Int. J. Mod. Phys. C 11, 1157 (2000); K. Sznajd-Weron, Acta Physica Polonica B 36, 2537 (2005).

[4] R.N. Costa Filho, M.P. Almeida, J.S. Andrade Jr., and J.E. Moreira, Phys. Rev. E 60, 1067 (1999); R.N. Costa Filho, M.P. Almeida, J.E. Moreira, and J.S. Andrade Jr., Physica A 322, 698 (2003).

[5] S. Fortunato and C. Castellano, Phys. Rev. Lett. 99, 138701 (2007).
[6] R. Albert and A.-L. Barabási, Rev. Mod. Phys. 74, 47 (2002); M.E.J. Newman, SIAM Rev. 45, 167 (2003); S. Boccaletti, V. Latora, Y. Moreno, M. Chavez, and D.-U. Hwang, Phys. Rep. 424, 175 (2006).

[7] S. Gil and D.H. Zanette, Phys. Lett. A 356, 89 (2006); P. Fronczak, A. Fronczak, and J.A. Hołyst, Phys. Rev. E 73, 046117 (2006); D. Stauffer, M. Hohnisch, and S. Pittnauer, Physica A 370, 734 (2006); P. Holme and M.E.J. Newman, Phys. Rev. E 74, 056108 (2006); J.M. Pacheco, A. Traulsen, and M.A. Nowak, Phys. Rev. Lett. 97, 258103 (2006); I.J. Benczik, S.Z. Benczik, B. Schmittmann, and R.K.P. Zia, Europhys. Lett. 82, 48006 (2008).

[8] H.-H. Jo, W.-S. Jung, and H.-T. Moon, Europhys. Lett. 73, 306 (2006); Phys. Rev. E 74, 026120 (2006).

[9] B. Latané and J.M. Darley, Am. Sci. 57, 244 (1969).

[10] L.A. Penner, J.F. Dovidio, J.A. Piliavin, and D.A. Schroeder, Annu. Rev. Psychol. 56, 365 (2005).

[11] S. Rosen, S.E. Mickler, and J.E. Collins II, J. Pers. Soc. 
Psychol. 53, 288 (1987).

[12] J.A. Piliavin, J.F. Dovidio, S.L. Gaertner, and R.D. Clark III, in Cooperation and helping behavior: Theories and research, edited by V.J. Derlega and J. Grzelak (Academic, New York, 1982).

[13] P.R. Amato, J. Pers. Soc. Psychol. 45, 571 (1983).

[14] R.V. Levine, T.S. Martinez, G. Brase, and K. Sorenson, J. Pers. Soc. Psychol. 67, 69 (1994).

[15] H. Hinrichsen, Adv. Phys. 49, 815 (2000); G. Ódor, Rev. Mod. Phys. 76, 663 (2004).

[16] For example, see C.W. Gardiner, in Handbook of Stochastic Methods, 2nd Ed. (Springer-Verlag, New York, 1985).

[17] There exists the exceptional region where $\rho_{\infty}$ for $b>0$ is larger than $\rho_{\infty}$ for $b=0$, which eventually disappears as soon as $b \geq \frac{1}{4}$. This region is surrounded by two lines and one curve: $c=\frac{3}{4} a+\frac{1}{4}, c=\left(\frac{1+2 b+\sqrt{1-4 b}}{2}\right) a+\frac{1-2 b-\sqrt{1-4 b}}{2}$, and $a=\frac{2 b c+b-2 c^{2}+3 c-1+\sqrt{(c-1-b)(c-1+3 b)}}{1+3 b-c+\sqrt{(c-1-b)(c-1+3 b)}}$.

[18] T.E. Harris, Ann. Prob. 2, 969 (1974).

[19] In finite systems with absorbing states, the system will be always trapped into one of absorbing states in the longtime limit. Thus, it is well known that the average over surviving samples is a useful quantity for the finite-size properties. Here, the order parameter $\rho$ is averaged over surviving samples only.

[20] M. A. Muñoz, Phys. Rev. E 57, 1377 (1998); M. A. Muñoz, F. Colaiori, and C. Castellano, Phys. Rev. E 72, 056102 (2005).

[21] M.H. Kim and H. Park, Phys. Rev. Lett. 73, 2579 (1994); W. Hwang, S. Kwon, H. Park, and H. Park, Phys. Rev. E 57, 6438 (1998). 